\title{
Bases para un proyecto de intervención didáctica en el medio urbano de Córdoba (educación primaria)
}

\begin{abstract}
Bases for a didactic intervention project in the urban environment of
\end{abstract} Cordova (primary education)

\author{
Dr. Miguel Jesús LÓPEZ SERRANO \\ Universidad de Córdoba \\ mjlopez@uco.es
}
Dr. Francisco VALVERDE FERNÁNDEZ Universidad de Córdoba
de1vafef@uco.es

\author{
Dr. Rafael GUERRERO ELECALDE \\ Universidad de Córdoba \\ rgelecalde@uco.es
}

\begin{abstract}
Resumen: En la ineludible tarea educativa de la difusión del patrimonio urbano, proponemos un método de trabajo para el conocimiento didáctico de la ciudad de Córdoba que, junto a unos supuestos sobre la organización de la trama urbana de la misma, se acompaña de una fundamentación didáctica que creemos que podría ser extrapolable y utilizable en el proceso educativo de difusión del patrimonio urbano de cualquier ciudad histórica.

Abstract: In the unavoidable educational task of the dissemination of urban heritage, we propose a working method for the didactic knowledge of the city of Córdoba that, together with some assumptions about the organization of its urban fabric, is accompanied by a didactic foundation that We believe that it could be extrapolated and usable in the educational process of disseminating the urban heritage of any historic city.
\end{abstract}

Palabra clave: Patrimonio, didáctica de la historia local, educación primaria, Ciencias Sociales.

Keywords: Heritage, didactics of local history, primary education, Social Sciences. 
Sumario:

I. Introducción.

II. Fundamentación teórica y metodológica.

2.1. Fundamentación científica.

2.2. Fundamentación didáctica.

III. Propuesta de realizaciones para el primer año de desarrollo del proyecto.

IV. Recursos materiales, técnicos, y económicos.

4.1. Recursos materiales y técnicos.

4.2. Aspectos administrativos.

V. Bibliografía.

Recibido: septiembre 2021 .

Aceptado: noviembre 2021. 


\section{INTRODUCCIÓN}

La ciudad es un hecho muy complejo al que contribuyen a explicar multitud de fenómenos de carácter histórico, geográfico, artístico, económico, político, social y de muy diversa índole.

El complejo entramado que constituye hoy el fenómeno urbano tiene un indudable valor patrimonial cuyo conocimiento enriquece al propio habitante de la ciudad, sea cual sea su naturaleza (edad, sexo, nivel cultural, situación socioeconómica, etc.) y a cualquier visitante.

De esa cualidad de la urbe como enriquecedora para su conocedor son conscientes las diversas instituciones que se ocupan de la ciudad y, por ello, en las últimas décadas, junto al proceso de recuperación de las ciudades para la vida del ciudadano, se viene desarrollando también una importante labor institucional de fomento del estudio sobre la ciudad entre sus diferentes habitantes y visitantes.

Esta corriente investigadora y de difusión del conocimiento de la ciudad viene siendo desarrollada muy en concreto por la institución municipal, aunque en muy diverso grado de desarrollo según los países. En general es apreciable en la oferta de muchos municipios una tendencia actual a considerar que dicha tarea no es específica de un área particular propia de la institución municipal (léase por ejemplo el área de Cultura) sino que debe ser una cuestión presente en la actuación de diversos frentes.

En Córdoba es indudable que se han hecho actuaciones en esta línea con resultados concretos muy diversos, pero creemos que todavía son muchas las tareas a realizar. En este sentido nuestro grupo de trabajo propone una línea de actuación que parte de unos determinados presupuestos.

\section{FUNDAMENTACIÓN TEÓRICA Y METODOLÓGICA}

\subsection{Fundamentación científica}

El proceso de difusión del conocimiento de la ciudad implica actuaciones puntuales y concretas. Estas deben enmarcarse en un programa global y amplio 
que responda a unos presupuestos teóricos fundamentados. En ese sentido, aun cuando puede ser objeto de análisis una parte de la ciudad, un barrio, una calle, una plaza, un monumento, un estilo artístico, un itinerario urbano, etc., pensamos que ese conocimiento parcial debe partir de una concepción global de la ciudad y en este caso de Córdoba. A este respecto, consideramos que cualquier acercamiento al conocimiento de nuestra ciudad debe estar enmarcado en la referencia de Córdoba como una realidad urbana compleja y extensa en el espacio, así como en el tiempo de su configuración. El devenir de los acontecimientos históricos ha estructurado en una realidad multiforme en la que se pueden señalar algunas líneas maestras en su conformación:

- La presencia de una clara dualidad morfofuncional entre el recinto intramuros de la ciudad (casco histórico) y la expansión "reciente" extramuros o periferia.

- La complejidad interna del recinto intramuros, el casco histórico o la ciudad tradicional, al haber funcionado a lo largo de un considerable período histórico como un recinto urbano doble: la Medina o Villa Alta y la Axerquía o Villa Baja.

- Esta propia complejidad del recinto intramuros se acentúa por la evolución morfológica y funcional de cada una de esas partes integrantes.

Así, en la Medina o Villa Alta su cuadrante noroccidental ha sufrido una amplia transformación reciente en su trama urbana por las llamadas operaciones de ensanches y transformaciones burguesas, que hacen actualmente dicha zona muy distinta de la parte suroccidental de la misma Medina, hoy identificada bajo el término de Judería. Del mismo modo, el papel jugado en siglos precedentes por el río Guadalquivir ha contribuido a configurar también determinados aspectos morfofuncionales de la parte meridional de la ciudad intramuros, tanto en la propia Medina como en la Axerquía.

La orla periférica de expansión extramuros de la ciudad tampoco tiene una uniformidad en su estructura urbana, por cuanto su proceso de configuración ha respondido a modos de asentamiento muy distintos. Así, puede hablarse de una expansión hacia el norte protagonizada por las clases sociales más altas, por las ventajas climáticas y medioambientales de la zona, la "Sierra", y materializada en una estructura urbana de características arquitectónicas de alto nivel, primero como área de segunda residencia de la población que la ocupaba y más reciéntemente, por los progresos de los medios de locomoción, como área de primera residencia o residencia permanente. Esta realidad adquiere nuevos matices cuando el acceso a la zona de las clases medias y el encarecimiento del suelo explican la 
disminución del tamaño de las parcelas de muchas de las edificaciones unifamiliares, la aparición actualmente de promociones de "adosados" y la presencia también en la zona de la edificación en altura.

Un desarrollo hacia el sur, al otro lado del Guadalquivir, zona tradicionalmente inundable por las crecidas del río y de mayor rigor climatológico, en la que son las clases menos favorecidas económicamente las que se instalan, en muchos casos mediante promociones urbanísticas de carácter benéfico o social.

Una ampliación hacia el oeste de las clases medias que, concebida originariamente como "ciudad jardín", por la presión especulativa de los años del boom urbanístico y del desarrollismo, se traduce en una estructura urbana de elevada edificación en altura, la mayor en la ciudad como conjunto, y de fuerte densidad edificatoria.

Una expansión hacia el este de clases desfavorecidas y media-baja y media que hacen de la zona un espacio urbano con una estructura mixta y compleja.

En cualquiera de los casos son cuatro líneas maestras de expansión, todas ellas susceptibles de ser matizadas y analizadas en mayor profundidad y consiguientemente zonificables con mayor detalle.

A esta estructura urbana compleja y con una ocupación humana con características muy diversas se añaden hoy a la ciudad de Córdoba unos problemas urbanos muy diversos, de entre los que señalamos algunos de los más evidentes:

- Los problemas de tráfico de la ciudad en su conjunto.

- En íntima conexión con el anterior, el proceso de recuperación del casco histórico y dentro de él la problemática de su peatonalización parcial o total.

- La recuperación del río y su entorno para su mayor incardinación en la trama urbana y su disfrute por el ciudadano como área de expansión, lúdica y cultural, potenciando sus valores ambientales.

- La problemática de la operación Renfe, que debe coordinar, en el proceso de ocupación del espacio liberado por el ferrocarril y sus instalaciones, los costes económicos y las necesidades ciudadanas. 
Esta ciudad así entendida pensamos que debe ser dada a conocer con toda su complejidad y problemática y desde una óptica comprometida, en el sentido de tener siempre presente que la conformación y vida de la propia ciudad se ha gestado (y produce) con conflictos y tensiones para la propia estructura y, lo que es más importante, para sus habitantes. Por lo tanto, el conocimiento de la ciudad y su difusión debe hacerse desde el análisis de la estructura física de la ciudad y de los problemas generados por y a sus habitantes.

En los momentos actuales, un primer acercamiento al conocimiento (y a su difusión) de la ciudad de Córdoba parece que no puede pasar por alto la reciente declaración de la misma como Patrimonio de la Humanidad. Dicha declaración afecta a parte de la ciudad histórica y aunque no podemos olvidar al resto del amplio recinto histórico cordobés ni al conjunto de la trama urbana cordobesa, incluidas sus áreas de expansión periférica, por cuanto es ese todo el que configura la ciudad real actual, tampoco podemos olvidar que esa zona está llamada a convertirse en la imagen más emblemática de la misma. Por ello creemos que debe partirse de ella, aunque con el objetivo de abordar sin solución de continuidad el estudio de la ciudad en su totalidad.

Desde estos presupuestos, dar a conocer la zona del casco histórico de Córdoba declarada Patrimonio de la Humanidad, independientemente de los procedimientos didáctico-pedagógicos con que abordemos esta tarea, creemos que debe abarcar los siguientes apartados:

Delimitación de la Zona y relaciones de la misma con el resto del entorno urbano. Razones para su delimitación. El proceso seguido para la obtención de la declaración.

Análisis de la trama urbana de la Zona. Características de la misma y semejanzas y diferencias respecto del resto de la trama urbana de la ciudad. Diferencias internas en la trama urbana de la Zona, posibilidades de delimitación de áreas diferenciadas internas.

Estudio de los edificios singulares ubicados en la Zona, desde la perspectiva de ser éste el espacio donde se ubican la mayor parte de los edificios y conjuntos arquitectónicos de carácter monumental que justifican la declaración de Patrimonio de la Humanidad.

Valoración del resto del caserío de la Zona, analizando también sus valores patrimoniales como exponente de la evolución urbana de la ciudad y de modos de vida todavía vigentes, aunque, en algunos casos, con disfunciones evidentes. 
Acercamiento al estado actual de la zona en cuanto al número, variedad, calidad y carencias de sus dotaciones. Este último apartado nos permite abordar el conocimiento del elemento humano que se asienta en la zona, conocimiento que puede organizarse desde los siguientes apartados:

- Estudio demográfico de la población de la Zona y diagnosis sobre su situación cuantitativa y cualitativa: número, evolución, composición. Detección de problemas.

- Análisis de las funciones que realiza esa población y también de las que demanda la misma. Este análisis funcional termina complementando el análisis morfológico de la Zona que hemos planteado en apartados anteriores y contribuye a presentar un panorama del estado actual del espacio desde el punto de vista humano y urbanístico, así como unas reflexiones sobre la posible potenciación del mismo.

- Todo lo anterior es una secuencia que debe llevar a valorar los problemas y contradicciones que caracterizan a esta zona de la ciudad y que podrían resumirse en:

- Envejecimiento demográfico, despoblación.

- Deterioro del caserío/necesidad de su recuperación.

- Terciarización de la zona.

- Carencia de dotaciones de ocio/esparcimiento.

- Problemas de circulación/peatonalización.

\subsection{Fundamentación didáctica}

En las ciudades habita un porcentaje cada vez más elevado de la población mundial. De hecho, la mayor parte de las experiencias y necesidades de los individuos se producen y satisfacen, mejor o peor, en medios urbanos. Las personas empleamos una cantidad muy importante de nuestro tiempo en conocer y utilizar la ciudad. Recorrer calles, utilizar servicios públicos, acudir a lugares de trabajo y de ocio, relacionarnos con otros individuos y grupos... conllevan una variedad de aprendizajes que realizamos consciente e inconscientemente en el medio urbano.

Sin embargo, abordar la enseñanza de la ciudad es una cuestión que no tiene respuestas del todo satisfactorias desde el punto de vista educativo. Si observamos la presencia del tema urbano en los currículos escolares, vemos que aparece aquí y allá como un factor explicativo de hechos y procesos históricos, económicos, 
energéticos, etc. En definitiva, resulta difícil adoptar un modelo de análisis que explique globalmente el funcionamiento de la ciudad. Posiblemente el enfoque ambiental proporciona el punto de vista más amplio para comprender, utilizar y mejorar la ciudad.

La ciudad no es un medio "natural", sino un medio "construido"; en consecuencia, el entorno físico urbano está determinado y definido socialmente.

En una ciudad podemos estudiar flujos de materia y energía, índices de contaminación atmosférica y acústica, tipología de las viviendas y su distribución, superficie y distribución de los espacios verdes... Pero todos estos aspectos sólo adquieren significado como manifestaciones de un ambiente urbano, resultado de un determinado sistema de organización social. Un enfoque ambiental de la ciudad debe tratar de explicar cómo se articulan las funciones sociales en el espacio urbano, cómo se reproduce esta organización en el entorno físico, de qué manera afecta todo lo anterior a la calidad de vida y qué intervenciones son necesarias sobre el conjunto del sistema urbano para mejorarlo.

Podernos partir de un cierto consenso sobre una serie de funciones sociales básicas que los grupos humanos llevan a cabo en el espacio en general, y en el espacio urbano de forma específica. Una posible selección de estas funciones sería: habitar, trabajar, recrearse, circular, establecer normas. Estas funciones sociales pueden actuar como inclusores de cualquier actividad que pueda interesarnos analizar en el espacio urbano y, en consecuencia, tienen un importante valor organizativo y sistematizador.

Por otra parte, podemos servirnos de lo que llamaremos "claves interpretativas", y que no son otra cosa que una serie de conceptos metodológicos que podemos usar para elaborar explicaciones en las ciencias sociales. Principios formulados en términos de pares opuestos, de los que seleccionamos aquí algunos que nos parecen más relevantes para el conocimiento z consenso/conflicto. Estas claves interpretativas actúan como bisagras sobre las que giran las funciones sociales señaladas más arriba.

El cruce de estos dos ejes -temático y metodológico- nos sirve de trama para extraer los principales problemas que afectan al medio urbano. Veamos un ejemplo: si pretendemos abordar la problemática de la vivienda en la ciudad -incluida en la función social de habitar-, los aspectos más relevantes a tratar serían las relaciones entre población y vivienda, el proceso de urbanización, la tipología de las viviendas y su distribución en el espacio urbano, los precios de venta y alquiler, las previsiones de los planes de ordenación urbana, etc. En este núcleo temático sobre la vivienda las claves "interpretativas" actuarían 
del modo siguiente: Las nociones de cambio/continuidad nos situarían delante de problemas como las transformaciones y permanencias de modelos arquitectónicos y sus consecuencias desde el punto de vista de la conservación o destrucción del patrimonio arquitectónico urbano; las ideas de semejanza/diversidad nos aproximarían a los contrastes del paisaje urbano y a los problemas de segregación; los principios de armonía/desequilibrio nos pondrían delante de problemas como el crecimiento incontrolado de ciertas zonas urbanas frente a la ruina y despoblación de otras; las nociones de consenso/conflicto nos acercarían a problemas como el de la escasez de viviendas y la especulación.

En los últimos años son cada vez más frecuentes las propuestas de estudio de la ciudad desde perspectivas interdisciplinares semejantes en varios aspectos a la que aquí hemos desarrollado. Un enfoque del estudio de la ciudad como el que acabamos de presentar condiciona de forma importante el proceso de enseñanza aprendizaje. La formulación ambiental no afecta sólo a los contenidos sino también a la manera de enseñarlos. Si pretendemos desarrollar en el alumnado las capacidades de observar de forma crítica el medio urbano, recoger y organizar datos sobre su funcionamiento, elaborar hipótesis interpretativas de los procesos que observa, y discutir con otros sus conclusiones, así como las propuestas para mejorar las condiciones del medio observado, debemos seleccionar el tipo de actividades, los recursos didácticos y las relaciones de comunicación que contribuyan de forma más directa a estos fines.

Debemos conceder un mayor valor formativo a aquellas actividades de enseñanza que favorezcan aprendizajes cognitivos, pero también experienciales, sobre el medio urbano. En este sentido, adquiere especial relevancia la apertura del aula al entorno urbano y a los recursos formativos del mismo. La ciudad en su conjunto se convierte así en una fuente información -y de formación- de primer orden. La labor del profesor consiste en planificar las condiciones para que los alumnos pongan en cuestión sus percepciones espontáneas y elaboren nuevas explicaciones más complejas y completas.

Sin pretender ser exhaustivos, señalamos algunos de los usos didácticos que resultan más indicados para trabajar sobre la ciudad desde una perspectiva ambiental:

- Observaciones y visitas ocasionales.

- Investigaciones singulares.

- Itinerarios urbanos.

- Simulaciones y juegos de rol.

- Talleres, campañas, concursos... 


\section{PROPUESTA DE REALIZACIONES PARA EL PRIMER AÑO DE DESARROLLO DEL PROYECTO}

Dentro del marco en el que concebimos a la ciudad de Córdoba y consecuentemente con nuestros presupuestos epistemológicos y metodológicos, nuestra propuesta de actuación para la difusión del conocimiento de la ciudad de Córdoba, y en una primera fase, de la Zona declarada Patrimonio de la Humanidad, se concretaría en el siguiente trabajo a realizar durante el primer año:

1. Elaboración de un material mediante el que se dé a conocer la Zona declarada Patrimonio de la Humanidad, analizando con rigor su delimitación y sus características generales como planteábamos anteriormente. Este material sería la base para la difusión del conocimiento de la Zona a diferentes colectivos: escolares y estudiantes de los diferentes niveles del sistema educativo, profesorado de los mismos, colectivos ciudadanos, visitantes, etc. Para cada uno de los colectivos destinatarios el material base se adecuará según nuestros presupuestos métodológicos y pedagógicos.

2. A partir de ese material base la concreción de nuestra tarea para el primer año se dirigiría al alumnado del Primer Ciclo de ESO y supondría:

2.1. Elaboración de una primera unidad didáctica sobre delimitación, localización y características de la trama urbana de la Zona y análisis de su caserío, diferenciando la significación de sus edificios singulares y no singulares. La unidad didáctica estaría formada por dos tipos de material complementario:

- Un cuaderno para el alumnado, donde se especificarían las actividades a realizar por el mismo, tanto dentro como fuera del aula, secuenciadas adecuadamente, y que iría acompañado del soporte gráfico, documental y estadístico que se estime conveniente.

- Una guía didáctica para el profesorado, donde se delimiten claramente aspectos como: Objetivos que se persiguen. Contenidos a desarrollar (en su triple vertiente: conceptual, procedimental y actitudinal) y relaciones con las diversas áreas del curriculum: especialmente con la de Ciencias Sociales, Geografía e Historia. Orientaciones metodológicas para el planteamiento de la unidad y el desarrollo de las actividades por parte del alumnado. Recursos didácticos útiles: bibliografía, material audiovisual, prensa... Criterios de evaluación que se pueden utilizar.

2.2. Seguimiento de la experimentación del material elaborado y evaluación posterior del proceso. 
2.3. La plaza como recurso didáctico. Este espacio ha sido el ámbito elegido por la ciudad para la ubicación en ella de todo un conjunto de elementos que perpetúan en la memoria las múltiples funciones que han venido definiendo la vida urbana: civil, religiosa, administrativa, comercial, institucional, etc. y todo ello mediante la ubicación en la plaza de un conjunto de elementos significativos de dichas funciones: monumentos, edificios de enorme singularidad artística y funcional, mobiliario, etc.

Desde el punto de vista físico, espacial, urbanístico, su cambio de escala respecto del otro elemento definidor del damero, la calle, la ha convertido en lugar de encuentro, de relación, de vida social, recogiendo la significación del ágora, foro, zoco, plaza mayor, en los distintos contextos.

En orden a estas consideraciones, creemos que aun cuando no podemos aislarla del resto del tejido urbano, se nos revela como un elemento importante para el conocimiento de la ciudad, por lo que la proponemos como centro para la realización de una serie de actividades: estudios monográficos, itinerarios, etc., que nos ayuden a conocer la complejidad de los fenómenos que conforman la realidad urbana.

Por lo común, en la plaza aparece un conjunto de elementos que pueden hacer reflexionar al estudiante sobre el complejo proceso de construcción de la ciudad.

La frecuente presencia en ella de algún edificio religioso (iglesia, convento, hospital, oratorio) permite no solo el análisis de los valores y características artísticas de éste, sino la consideración de la Iglesia como uno de los agentes que configuran el espacio urbano, por cuanto, en ocasiones, la plaza surge para dar prestancia a dicho edificio o para realizar en ella las funciones emanadas del mismo.

Las casas señoriales y de las clases más acomodadas que también abundan en las plazas, permiten, además del conocimiento de las características constructivas y ornamentales de la arquitectura civil, poner de manifiesto el papel que dichas clases han desempeñado también en el diseño de la trama urbana, pues, como en el caso de los edificios religiosos, también algunas plazas se abrieron por las necesidades que demandaban dichas casas o palacios: acceso, servicios, deseo de ostentación.

Igualmente, pueden aparecer edificios administrativos e institucionales: ayuntamientos, mercados, pósitos, cárceles. Las mismas consideraciones que para la Iglesia y las clases altas, pueden hacerse respecto de las instituciones 
como ordenadoras del proceso de urbanización de la ciudad y constructoras de edificios, en algunos casos, de indudable valor artístico.

De igual modo, y junto a esos diversos edificios singulares, en la plaza es frecuente encontrar vestigios de la arquitectura pasada popular que deben ser también objeto de análisis.

Desde el punto de vista funcional, escenario de la vida de relación de los ciudadanos, ha sido lugar de estancia. Tras la invasión de la ciudad por la circulación rodada, en determinadas plazas, se ha separado su espacio en dos ámbitos, el de estancia y el de circulación. Por lo común, a aquel se le ha concedido mayor prestancia mediante un variado mobiliario: bancos, farolas, jardines, parterres, fuentes, monumentos, estatuas, etc. El análisis de estos hechos puede propiciar en el estudiante la reflexión sobre el papel simbólico y representativo que la plaza ha desempeñado, y le permite ponerse en contacto con el estudio de aspectos históricos, literarios, científicos, etc.

También la plaza recoge o ha recogido un conjunto de actividades significativas: comerciales, artesanales, festivas, de conmemoración de acontecimientos políticos y religiosos, etc. Estos hechos, visibles o no en los elementos físicos de ella, deben ser igualmente analizados, incluso, si es posible, poniendo en contacto al estudiante con las propias fuentes.

En general, las plazas a que venimos haciendo referencia tienen una relativa importancia espacial y un cierto porte arquitectónico o constructivo. Pero junto a éstas, existen también otras plazas, generalmente pequeñas, cruces o ensanches de calles, adarves, etc., que con el cambio de escala operado en la ciudad han perdido su significación y funciones. Creemos no obstante que deben también ser analizadas para evidenciar el proceso evolutivo de construcción de la ciudad.

Del mismo modo, tendría también que transmitirse al alumno que la plaza puede surgir como resultado de, al menos, dos procesos: o por acumulación paulatina y anárquica de los elementos de la trama urbana a través del tiempo, o como resultado de un planteamiento urbanístico puntual tendente a la creación ex profeso de la misma con la consiguiente huella en su morfología.

Estas y otras reflexiones que pudieran hacerse nos parece que justifican el aprovechamiento didáctico de la plaza para el acercamiento al conocimiento del patrimonio urbano.

Tras las consideraciones teóricas precedentes sobre el valor didáctico de la plaza como un método posible para el conocimiento del patrimonio urbano, 
aplicándolo al caso cordobés hemos elegido una plaza concreta para constatar las posibilidades didácticas que ellas nos brindan.

La plaza seleccionada, la del Corazón de María, creemos que por sus características morfológicas y funcionales cubre un amplio espectro de esas posibilidades.

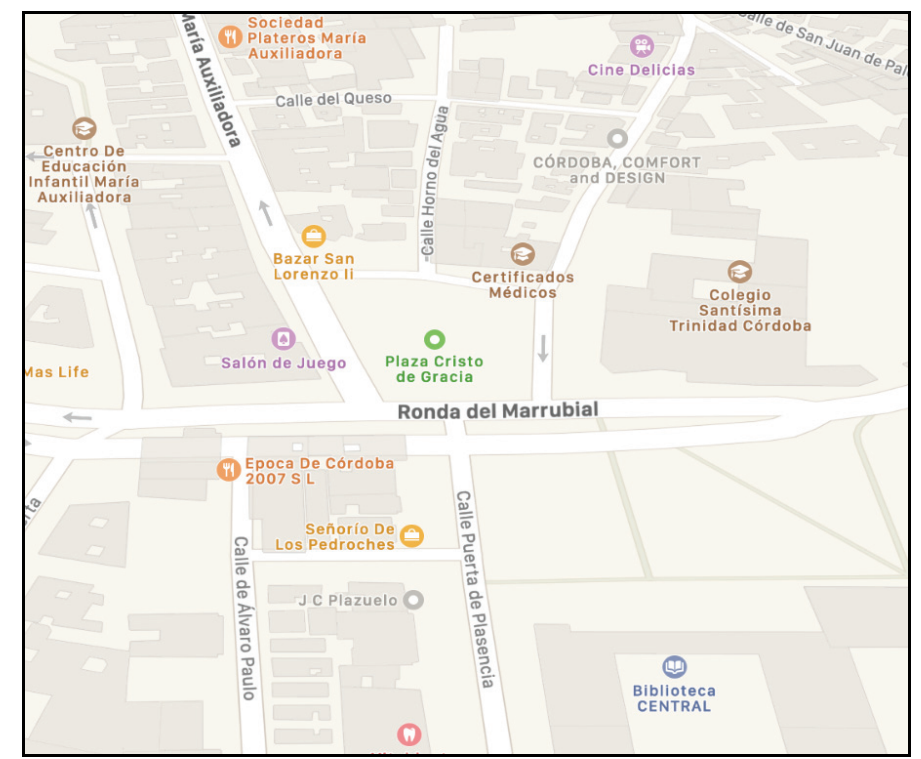

Figura 1. Ubicación Plaza del Corazón de María.

Fuente: Google Maps

En primer lugar, ofrecemos una información sobre ella, para aprovechamiento del profesor, y como último punto presentamos una propuesta de organización y desarrollo de la visita a la plaza, propuesta que no pretende ser otra cosa que un conjunto de orientaciones de tipo general para que sirvan de guía al profesor y que éste, de acuerdo con el nivel psicopedagógico de sus alumnos y de los objetivos que se haya marcado, las seleccione y desarrolle según sus necesidades docentes.

Como cuestión, posiblemente previa, habría que plantearse alguna/s sesión/s de trabajo con el profesorado implicado, para presentar y contextualizar el material didáctico a utilizar.

La experimentación propiamente dicha de este material conllevaría la puesta en funcionamiento de una mínima infraestructura material y humana (monitores), 
que hiciera posible el contacto con los centros, la organización del trabajo de campo previsto en las actividades del alumnado, la entrega y recogida de material...

La evaluación de los materiales elaborados y del proceso seguido en su experimentación supondría la utilización de técnicas standard: cuestionarios y entrevistas al profesorado y al alumnado, opiniones de monitores y técnicos municipales...

\section{RECURSOS MATERIALES, TÉCNICOS, Y ECONÓMICOS}

\subsection{Recursos materiales y técnicos}

Los necesarios para la puesta en marcha de esta idea son básicamente los existentes en los Departamentos universitarios donde está integrado el profesorado antes citado. En este sentido, no conllevan ningún presupuesto específico. Es importante señalar, sin embargo, que para el desarrollo del proyecto resulta sustancial que el equipo pueda disponer de la información y documentación municipal que sea de interés para el mismo (tanto cartográfica, como estadística o de otro tipo), considerándose de especial relevancia la correspondiente al Plan Especial del Casco Histórico.

Igualmente habría que decidir si se imputan como gastos para el desarrollo del proyecto los derivados de algunos trabajos técnicos especializados (por ejemplo, delineación, fotografías, dibujos...) necesarios como apoyo gráfico de los materiales, así como la edición e impresión del conjunto de material didáctico elaborado.

\subsection{Aspectos administrativos}

Sugerimos que la fórmula administrativa más adecuada para la puesta en marcha del proyecto que aquí se esboza es la firma de un convenio de colaboración entre el Ayuntamiento de Córdoba y la Universidad (Facultad de Ciencias de la Educación), en el que se detallen los compromisos y aportaciones de ambas partes, las fórmulas previstas para su desarrollo, la duración del mismo (puede ser un año, renovable por períodos similares con el acuerdo mutuo) y los mecanismos de seguimiento que se consideren adecuados. 


\section{BIBLIOGRAFÍA}

- AGUADO MOLINA, M., "Aportaciones de la museología al aprendizaje de los futuros maestros de E. Infantil sobre Didáctica específica de las Ciencias Sociales", en LÓPEZ TORRES, E; GARCÍA RUIZ, C. R., y SÁNCHEZ AGUSTI, M. (Eds.), Buscando formas de enseñar: investigar para innovar en la didáctica de las Ciencias Sociales. Universidad de Valladolid, 2018, pp. 233-242.

- ÁVILA, R. M., y DUARTE, O. "Mi patrimonio, mi museo". Una experiencia didáctica en la formación inicial del profesorado de Educación Infantil, en PAGÈS, J. Y A. SANTISTEBAN, A. (Coord.), Una mirada al pasado y un proyecto de futuro: investigación e innovación en didáctica de las ciencias sociales. AUPDCS, 2014.

- CUENCA LÓPEZ, J.M., ESTEPA JIMÉNEZ, J., MARTÍN CÁCERES, M.J.: «El patrimonio cultural en la educación reglada». Patrimonio cultural de España, N. ${ }^{\circ} 5$ de 2011.

- FUERTES MUÑOZ, C., "Emoción y patrimonio. Educación emocional y fuentes orales en el diseño de itinerarios didácticos", en Didácticas Especificas, N. ${ }^{\circ} 15(2016)$.

- GARCÍA LUQUE, A., “Aprender el patrimonio con perspectiva de género", en Boletín de la Sociedad de Amigos de la Cultura de Vélez- Málaga, $\mathrm{n}^{\circ} 7$ (2016).

- GARCÍA-MORÍS, R., "Propuesta metodológica para el uso de las fuentes históricas demográficas de la Edad Moderna como recurso didáctico", en Revista de Didácticas Específicas, núm. 14 (2016) 71-85.

- IBÁÑEZ-ETXEBERRIA, A.; FONTAL MERILLAS, O., y RIVERO GRACÍA, P., "Educación patrimonial y TIC en España: marco normativo, variables estructurantes y programas referentes", en Arbor: Ciencia, pensamiento y cultura, vol. 194, n. ${ }^{\circ} 788$ (2018).

- JIMÉNEZ-ESQUINAS, G., 'De 'añadir mujeres y agitar' a la despatriarcalización del patrimonio: la crítica patrimonial feminista", en Revista $P H$ Instituto Andaluz del Patrimonio Histórico, $n^{\circ} 89$ (abril de 2016).

- LÓPEZ SERRANO, M.J., y GUERRERO ELECALDE, R., "El Museo Arqueológico de Córdoba para trabajar la historia local en Educación Primaria", en REIDICS. Revista de Investigación en Didáctica de las Ciencias Sociales, 7 (2020) 153-170. 
- OLAVE FARÍAS, D., "El itinerario didáctico: una propuesta metodológica para el análisis geo-histórico local", en Geo-enseñanza, 10 / 2 (2005) 197-208. Disponible en línea: https://www.redalyc.org/articulo.oa?id=36010206.

- PAGÈS, J., "La formación inicial del profesorado para la enseñanza del patrimonio histórico y de la historia", en Treballs d'Arqueologia, núm. 6 (2000) 205-217.

- PEDREÑO ROS, D. Aportaciones Didácticas de la Historia Local y Familiar al Proceso de Enseñanza-Aprendizaje de las Ciencias Sociales. Experiencias en Tres Centros Educativos de la Región de Murcia de Educación Primaria, Secundaria y PCPI. Tesis defendida en la Universidad de Murcia, 2015: http://hdl.handle.net/10201/46981.

- POBLET ROMEU, M.J., "El patrimonio histórico, una herramienta para la enseñanza de la historia, en Iber: Didáctica de las ciencias sociales, geografia e historia, núm. 40 (2004) 116-122.

- PRATS, J., "El estudio de la Historia local como opción didáctica. ¿destruir o explicar la historia", en Iber Didáctica de las ciencias sociales, geografia. e historia, núm. 8 (abril 1996).

- RICO CANO, L.., y ÁVILA RUIZ, R.M., "Difusión del Patrimonio y educación. El papel de los materiales curriculares. Un análisis crítico", en BALLESTEROS, E. et alii (coords.), El Patrimonio y la Didáctica de las Ciencias Sociales. Universidad de Castilla-La Mancha, Ediciones de la Universidad de CastillaLa Mancha Asociación Universitaria de Profesores de Didáctica de las Ciencias Sociales, 2003, pp. 31-40.

- RIVERO, P. (2008). "Propuesta para una integración del patrimonio arqueológico en el Nuevo currículo de Educación primaria", en ÁVILA, R.M.; CRUZ, A, Y M.C. DÍEZ, M.C. (Eds.), Didáctica de las Ciencias Sociales, currículo escolar y formación del profesorado. La didáctica de las Ciencias Sociales en los nuevos planes de estudio, Universidad de Jaén / Asociación universitaria de profesorado de Didáctica de las Ciencias Sociales, 2008, pp. 391-400.

- SERRANO MORAL C., Otra educación en los museos es posible. Propuesta de un proyecto colaborativo desde la enseñanza de las ciencias sociales. Tesis doctoral. Universidad de Málaga, 2018. 